\title{
APLICABILIDADE E BENEFÍCIOS DE SOFTWARES E SIMULADORES EM PROCESSOS DE ENSINO-APRENDIZAGEM ${ }^{1}$
}

\author{
APPLICABILITY AND BENEFITS OF SOFTWARE AND SIMULATORS IN TEACHING \\ LEARNING PROCESSES
}

\section{POTENCIALIDAD Y BENEFICIOS DEL SOFTWARE, SIMULADORES Y SUS PROCESOS DE APRENDIZAJE}

\section{Marcelo Salvador Celestino²; Vânia Cristina Pires Nogueira Valente 3}

\begin{abstract}
RESUMO
O objetivo deste artigo foi o de identificar a aplicabilidade e benefícios de softwares, processos de simulação e simuladores para a prática educacional em diferentes contextos. O texto aqui apresentado integrou a fundamentação teórica e viabilizou a dissertação de mestrado intitulada "Aplicabilidade de softwares de simulação para o ensino de Tomografia Computadorizada para técnicos e tecnólogos em Radiologia". A literatura consultada evidencia a aplicabilidade da simulação e suas vertentes em diferentes áreas, como gestão, indústria, automobilística, militar, Saúde, dentre outras, com enfoque educacional. Os benefícios descritos na literatura referem-se à aquisição de habilidades, ao desenvolvimento de competências, à repetição exaustiva de uma atividade sem prejuízos ou riscos a pacientes, ao baixo custo, dentre outros, por meio de softwares e simuladores, e salientam o uso destes como ferramentas de apoio ao docente para o ensino-aprendizagem.
\end{abstract}

PALAVRAS-CHAVE: Educação. Software. Técnicas de Simulação. Educação pelas mídias. Tecnologia.

\begin{abstract}
The aim of this paper was to identify the applicability and benefits of softwares, simulation processes and simulators for the educational practice in different contexts. The text presented here integrated the theoretical foundation and made possible the master's dissertation entitled "Applicability of simulation software for the teaching of Computed Tomography for technicians and technologists in Radiology". The consulted literature shows the applicability of the simulation and its aspects in different areas, such as management, industry, automotive, military, health, among others, with educational focus. The benefits described in the literature refer to the acquisition of skills, the development of skills, the exhaustive repetition of an activity without harm or risk to patients, the low cost, among others, through software and simulators, and emphasize its use as tools to support the teacher for teaching-learning.
\end{abstract}

KEYWORDS: Education. Software. Simulation Techniques. Media Education. Technology.

\footnotetext{
${ }^{1}$ Esse trabalho é parte da fundamentação teórica de uma dissertação de mestrado.

${ }^{2}$ Mestre em Mídia e Tecnologia - Universidade Estadual Paulista Júlio de Mesquita Filho (UNESP). Bauru, SP Brasil. Tecnólogo em Radiologia. E-mail: marcelo.salvador@unesp.br

${ }^{3}$ Doutora em Engenharia Civil - Escola Politécnica da Universidade de São Paulo (USP). São Paulo, SP - Brasil. Professora Doutora Associada - Universidade Estadual Paulista Júlio de Mesquita Filho (UNESP). Bauru, SP Brasil. E-mail: vania.valente@unesp.br

Submetido em: 07/02/2020 - Aceito em: 12/05/2020
}

(C) ETD-Educação Temática Digital $\quad$ Campinas, SP $\quad$ v.23 $\quad$ n.4 $\quad$ p.881-903 $\quad$ out./dez. 2021




\section{RESUMEN}

El objetivo de este trabajo fue identificar la aplicabilidad y los beneficios de softwares, los procesos de simulación y los simuladores para la práctica educativa en diferentes contextos. El texto presentado aquí integró los fundamentos teóricos y permitió la disertación de maestría titulada "Aplicabilidad del software de simulación para la enseñanza de la tomografía computarizada para técnicos y tecnólogos en Radiología". La literatura consultada muestra la aplicabilidad de la simulación y sus aspectos en diferentes áreas, tales como administración, industria, automotriz, militar, salud, entre otras, con enfoque educativo. Los beneficios descritos en la literatura se refieren a la adquisición de habilidades, el desarrollo de cualificaciones, la repetición exhaustiva de una actividad sin daño o riesgo para los pacientes, el bajo costo, entre otros, a través de software y simuladores, y enfatizan el uso de estos como herramientas para apoyar al cuerpo docente para la enseñanza-aprendizage.

PALAVRAS-CLAVE: Educacion. Software. Técnicas de Simulación. Educación en médios. Tecnología.

\section{INTRODUÇÃO}

A simulação permite estudar sistemas complexos da vida real por intermédio de análises de modelos realistas, a fim de obter informações sobre o seu funcionamento e testar suposições para resolução de problemas (LAW; KELTON, 1991). Ela pode ser física ou digital/virtual, de maneira que os simuladores e os softwares de simulação computacional podem ser compreendidos como objetos capazes de reproduzir digitalmente uma atividade, com a finalidade de preparar uma pessoa para eventos reais (CLAYTON; GIZELIS, 2005; PAZIN FILHO; SCARPELINI, 2007; McHANEY, 2009; SADIDEEN et al., 2012).

$\mathrm{Na}$ área da Educação, a literatura demonstra que diversas disciplinas estão investindo nos processos didáticos que envolvem simulação, com o intuito de facilitar a integração e a aplicação de conteúdos teóricos na prática (CLAYTON; GIZELIS, 2005). Isso ocorre, possivelmente, porque os simuladores são objetos que permitem a comunicação e a interação entre estudantes e professores (ANDRADE, 2012), e aproximam a vivência dentro da sala de aula com a realidade de um mundo em que experimenta-se um notável crescimento nas pesquisas com softwares (MANOVICH, 2013). Destaca-se aqui a geração de estudantes chamados de "nativos digitais", que aprendem a respeito de aparatos tecnológicos usando-os no cotidiano, que preferem jogos a leituras de textos e livros, e que têm amplas habilidades e capacidade de aprendizagem por meios digitais e tecnológicos (PRENSKY, 2001; 2012).

Os softwares e/ou simuladores integram objetos inseridos em um contexto de metodologias ativas que, para Berbel (2011), podem ser entendidas como uma forma de representação por meio da realidade ou simulação de atividades presentes em nossa prática social, independente da área. Ainda de acordo com a autora, tais metodologias possibilitam uma forma de aprendizagem em que ocorrem a participação e engajamento dos alunos com inserção de novos elementos promotores de reflexão e discussão.

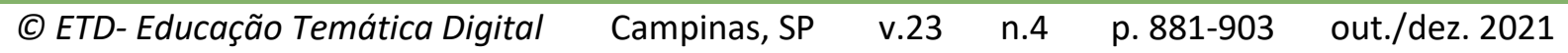


O trabalho de Fitzsimons (2014) também valoriza o processo de autorreflexão dos estudantes após a conclusão de um processo. O autor demonstrou pontos importantes de aprendizagem por meio da aplicação de metodologia ativa, dentre os quais destacam-se: a comunicação, o trabalho em grupo e a construção de uma rede de contatos.

Para a fundamentação da dissertação a que este trabalho se propôs, foram selecionadas as metodologias ativas por se tratar de uma alternativa pedagógica consolidada em diferentes áreas capazes de trazer uma abordagem inovadora para o ensino da Tomografia Computadorizada (TC), bem como outros conteúdos, no universo dos estudantes de cursos profissionalizantes e tecnológicos na área da Radiologia. Este meio é permeado por três pontos relevantes para o contexto da pesquisa: 1) pelas tendências pedagógicas tradicional e tecnicista; 2 ) pela compreensão e entendimento do atual cenário em que Educação e Tecnologias (digitais e midiáticas) fundem-se cada vez mais, originando a necessidade da busca por propostas metodológicas inovadoras, centradas nas tecnologias emergentes e nas metodologias ativas de ensino, focadas no estudante como sujeito central e autônomo da sua aprendizagem; 3) disponibilidade de softwares gratuitos que poderiam ser adaptados para a abordagem do ensino de TC, o que caracteriza, neste contexto, uma alternativa de fácil implementação e baixo custo.

O objetivo primário deste trabalho foi o de revisar conceitos e aplicações de softwares, simuladores e processos de simulação digitais em diferentes contextos sob a óptica da Educação, a fim de compor a fundamentação teórica da dissertação de mestrado intitulada "Aplicabilidade de softwares de simulação para o ensino de Tomografia Computadorizada para técnicos e tecnólogos em Radiologia" (CELESTINO, 2019). A literatura evidenciou os benefícios da aplicabilidade de softwares e simuladores em diferentes contextos e processos de ensino-aprendizagem, além da potencialidade destes enquanto ferramentas de apoio ao docente.

A estrutura do trabalho se divide em 3 partes: Método, em que é apresentado o panorama geral da pesquisa, forma de coleta e tratamento dos dados; Simulação: da imitação à prática, cujas subseções abordam pontos como revisão conceitual, histórico dos simuladores, a simulação por intermédio de softwares e recursos digitais, trabalhos correlatos e os benefícios descritos na literatura; Discussão, em que são apresentadas as inferências reflexivas dos autores sobre os principais pontos selecionados da revisão de literatura. Em seguida, são apresentadas a conclusão e as referências do trabalho. 


\section{MÉTODO}

Foram realizadas uma pesquisa exploratória e uma revisão sistematizada que ocorreram em três repositórios: NCBI (National Center for Biotechnology Information, em Português Centro Nacional para Informação Biotecnológica) (PUBMED, 2017), Science Direct (2017) e portal de periódicos da CAPES (PORTAL, 2018), cujo período de publicações foi ampliado de acordo com os desdobramentos da pesquisa, englobando referências de 1991 a 2020, envolvendo também sites, o que delineou a pesquisa como exploratória de natureza básica (FREGONEZE et al., 2014). Como complemento do artigo, foram realizadas: uma pesquisa por meio da ferramenta de pesquisa Google Acadêmico, a fim de localizar trabalhos cuja abordagem evidenciasse a aplicação de softwares ou simuladores em processos de ensino-aprendizagem em diferente áreas, dos quais foram selecionados 18 exemplos, após sua leitura e verificação de sua aderência ao tema deste trabalho; uma pesquisa por meio de um formulário Google Forms, intitulada "O uso de softwares e simuladores em processos de ensino-aprendizagem", a fim de coletar exemplos de instituições que utilizem tais objetos em práticas de ensino.

Os trabalhos foram selecionados pela sua adesão ao problema de pesquisa, cujo delineamento partiu da pergunta "De que maneira os softwares e técnicas de simulação, e/ou simuladores poderiam contribuir para o ensino da Tomografia Computadorizada (TC) nos cursos de Educação Profissional e Tecnológica em Radiologia?". Sendo a Radiologia uma especialidade médica e técnico-operacional da Saúde, e a pesquisa correlacionada em três áreas diferentes - Educação, Saúde e Mídia e Tecnologia - a tônica para o desenvolvimento deste artigo recai como sendo o tema central: "A Educação por meio de simuladores e/ou softwares de simulação", e tem como objetivo compreender o cenário de aplicação de simuladores, softwares e processos de simulação em geral, enquanto objeto de aprendizagem, em diferentes contextos, e viabilizar o desenvolvimento da dissertação de mestrado a qual a presente pesquisa integra parte da fundamentação teórica.

Após a seleção inicial do material de referência, foi realizada a caracterização dos descritores na Biblioteca Virtual em Saúde (BVS, 2018) em Descritores em Ciências da Saúde (DeCS), sendo escolhidos os termos Educação Profissionalizante, Educação Tecnológica, Educação em Saúde, Software e Simulação. Os critérios de inclusão dos trabalhos selecionados para análise foram: a) estar escrito nos idiomas Português, Inglês ou Espanhol; ser arquivo de revisão ou pesquisa; conter no título os termos simulation, simulador, software, training e Radiology ou Radiological ou termos correlatos, como PACS e medical imaging; b) ser software utilizado como ferramenta no ensino da TC ou conteúdo relacionado à disciplina/modalidade; c) conter no título palavras de alusão ao tema pesquisado.

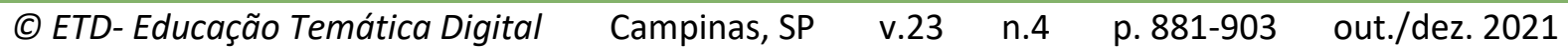


Na busca realizada no site da CAPES (PORTAL, 2018) o critério foi: ser avaliado pela CAPES com Qualis A1, A2 ou B1, na área de classificação Interdisciplinar, na classificação dos periódicos de triênio de 2013-2016. Foram considerados como critérios de exclusão os seguintes itens: a) aplicação de questionários (surveys), sem uma aplicação prévia de alguma técnica de simulação; b) uso de simulação que não fosse para fins educativos e temas relacionados à ultrassonografia (USG) e radioterapia (RT), por se tratar de métodos de diagnóstico e tratamento peculiares e distintos da TC; c) softwares de simulação com aplicabilidade em outras áreas; d) não possuir link para download. O tratamento de dados se deu de forma qualitativa. Para o corpus deste trabalho, excluíram-se os resultados específicos da área da Radiologia, já que estes serão abordados em outro artigo. A pesquisa completa está disponível na dissertação de Celestino (2019).

\section{SIMULAÇÃO: DA IMITAÇÃO À PRÁTICA}

Enquanto o simulacro pode ser entendido com uma cópia de algo real, que não atinge a perfeição, a simulação é uma técnica de aprendizagem baseada na representação de uma tarefa que existe na realidade (BAUDRILLARD, 1991). O termo simulacro foi introduzido por Jean Baudrillard (1991) em uma discussão contemporânea sobre o real e o imaginário, em que se aborda as relações entre a simulação e a representação. As três categorias de simulacros conceituadas por Baudrillard (1991, p. 151) são:

\footnotetext{
- simulacros naturais, naturalistas, baseados na imagem, na imitação e no fingimento, harmoniosos, otimistas e que visam a restituição ou a instituição ideal de uma natureza à imagem de Deus

; - simulacros produtivos, produtivistas, baseados na energia, na força, na sua materialização pela máquina e em todo o sistema da produção - objetivo prometiano de uma mundialização e de uma expansão contínua, de uma libertação de energia indefinida (o desejo faz parte das utopias relativas a esta categoria de simulacros); - simulacros de simulação, baseados na informação, no modelo, no jogo cibernético - operacionalidade total, hiper-realidade, objetivo de controle total. (BAUDRILLARD, 1991, p.151).
}

Tais conceitos são bastantes próximos e, geralmente, remetem à imitação do real e da prática. O quadro 1 os apresenta a partir de diferentes autores sobre simulação e simuladores, exibidos em ordem cronológica de publicação, em complemento aos autores citados anteriormente na Introdução deste artigo. 
QUADRO 1. Conceitos de simulação

\begin{tabular}{|c|l|}
\hline $\begin{array}{c}\text { Autores } \\
\text { Maran e Glavin (2003) }\end{array}$ & $\begin{array}{l}\text { Conceitos } \\
\text { Técnica educacional interativa e imersiva, que permite erros e } \\
\text { leva ao domínio técnico sem prejuízo aos pacientes. }\end{array}$ \\
\hline Ziv et al. (2006) & $\begin{array}{l}\text { Educação crítica e ética que preservam os pacientes de riscos } \\
\text { associados. }\end{array}$ \\
\hline $\begin{array}{c}\text { Pazin Filho e Scarpelini } \\
\text { (2007) }\end{array}$ & $\begin{array}{l}\text { Técnica de preparo profissional, cujo contato inicial ocorre por } \\
\text { meio da prática. }\end{array}$ \\
\hline Hillesheim e Schottz (2009) & $\begin{array}{l}\text { Modelo computacional que permite se obter inferências e } \\
\text { conclusões sobre o mundo real, por meio da lógica e estatística } \\
\text { em múltiplas áreas do conhecimento. }\end{array}$ \\
\hline Vidal-Gomel e Fauquet- & $\begin{array}{l}\text { Ferramenta que permite a visualização dinâmica de um } \\
\text { sistema. }\end{array}$ \\
\hline Alekhine (2016) & $\begin{array}{l}\text { Artefatos que simulam o comportamento técnico de um } \\
\text { sistema. }\end{array}$ \\
\hline
\end{tabular}

Fonte: os autores (2018)

Faz-se importante ter clara a diferença entre processos de simulação, que representam uma atividade ou situação, e os jogos digitais ou os serious games (jogos sérios), que são construídos sobre enredos, histórias, personagens e cenários, também utilizados em processos de ensino e aprendizagem para o desenvolvimento de competências e habilidades.

\subsection{Breve histórico e contexto}

Os simuladores tiveram suas relações iniciais para fins militares na Primeira Guerra Mundial, quando foram desenvolvidos com interesse para o treinamento de pilotos, na intenção de que eles adquirissem certa experiência e prática, antes do contato com um avião real. Isto ocorreu, basicamente, na década de 1950, com o desenvolvimento de objetos por meio da linguagem de programação conhecida por Fortran (BALADEZ, 2009). Na década de 1960 surgiu a linguagem GPSS (Global Purpose Simulation System, Sistema de Simulação de Propósito Global) em uma linguagem utilizada pela IBM para analisar sistemas complexos, com posterior desenvolvimento para as indústrias automobilística e geral, a partir da década de 70 (McHANEY, 2009). Já na Segunda Guerra Mundial, a simulação evoluiu para o uso de computadores, como explica Baladez (2009) a respeito dos computadores Mark I e ENIAC, cuja finalidade era realizar cálculos balísticos para a marinha e para o exército, respectivamente, na simulação do lançamento de mísseis. 
Clayton e Gizelis (2005) referem que a simulação encontra aplicação em diferentes áreas, sendo um método que trabalha, dentre outros aspectos, a transferência de habilidades, desta forma, preparando os estudantes e profissionais para o melhor desempenho de suas atividades. Segundo os autores (2005, p. 6):

[...] profissões como Direito, Trabalho Social, Planejamento, Política e Cuidados de Saúde usam alguma forma de simulação para desenvolver, praticar e testar a capacidade dos alunos de se comunicar, discutir e negociar com os outros de uma maneira que aplique ideias teóricas em um sentido prático. Nesse sentido, as habilidades desenvolvidas são altamente transferíveis, como se comunicar e negociar efetivamente com os outros são habilidades essenciais para a vida. (CLAYTON; GIZELIS, 2005, p. 6). (tradução livre)

Na área da Saúde, a chamada simulação realística tem sido utilizada para preparar os estudantes para o contato inicial da prática profissional. Este tipo de abordagem, cujo enfoque é ativo na construção do conhecimento, permite aos estudantes uma participação na problematização e busca de soluções para adversidades e problemas que possam permear a futura atuação profissional (FLORES; BEZ; BRUNO, 2014).

Diversos instrumentos têm sido utilizados no processo de simulação realística, como por exemplo, os manequins, os bonecos e as peças anatômicas, úteis ao treinamento de procedimentos técnicos específicos, como punção de acessos venosos, tendo como vantagem a possibilidade de repetição do processo, sem prejuízo a um paciente real (PAZIN FILHO; ROMANO, 2007; GEERAERTS; TRABOLD, 2011).

Tais bonecos e outros objetos aplicados na Educação em Saúde são cada vez mais sensíveis e possuem alta fidelidade, permitindo experiências gradativamente mais realísticas devido à interação com audiovisuais e com o toque, para o desenvolvimento e treinamento de habilidades em uma série de procedimentos manuais e, inclusive, avaliações e dinâmicas em grupo (CHETLEN, et al., 2015).

\subsection{A simulação por intermédio de recursos digitais}

Os softwares e os simuladores digitais estão inseridos em um contexto em que, segundo Straubhaar e LaRose (2004), as relações de comunicação e acesso à informação têm se expandido e redesenhado a sociedade como um todo, com ênfase para as relações digitais. A simulação digital, embora seja uma modalidade interativa artificial, proporciona um grau de imersão e atenção por parte do seu usuário, envolvendo diversos sentidos que permitem certa vivência e compreensão de uma determinada tarefa ou situação. Para ele, tanto a imersão, quanto o contexto dos simuladores, são relacionados ao grau de realidade com que os objetos e situação são representados (BORBA, 2014). 
No âmbito da Educação, os softwares de simulação, simuladores e processos de simulação em geral têm sido estudados como métodos ativos de aprendizagem. Estes proporcionam uma imersão na atividade proposta e podem ser vistos como recursos atrativos para a reprodução de tarefas e situações, pelo fato de representar uma atividade real ou caso fictício relacionadas ao ambiente profissional, o que permite aos estudantes observarem, ainda que de maneira digital, situações que poderão ocorrer em sua prática de estágio, ou no mercado de trabalho (BORBA, 2014; FLORES; BEZ; BRUNO, 2014; FAUQUET-ALEKHINE; PEHUE, 2016).

Como exemplos de simulação na prática, têm-se as escolas de aviação que oferecem cursos (AEROCLUB DE BAURU, 2018), treinamentos de voo (EJ ESCOLA DE AVIAÇÃO CIVIL, 2015) e treinamento de voo por instrumentos, por meio de softwares validados pela Agência Nacional de Aviação Civil (ANAC) e Administração Federal de Aviação (FAA Estados Unidos), como anuncia o site da AEROCON (2017).

A figura 1 é de um simulador de voo de uma nave Cessna Grand Caravan C208, utilizado por aeroclubes para o treinamento de novos pilotos. Nele, os botões e indicadores são cópia de uma aeronave real e são integrados com um aplicativo, que pode ser o Flight Simulator ou Prepar3D (HAMANN, 2012).

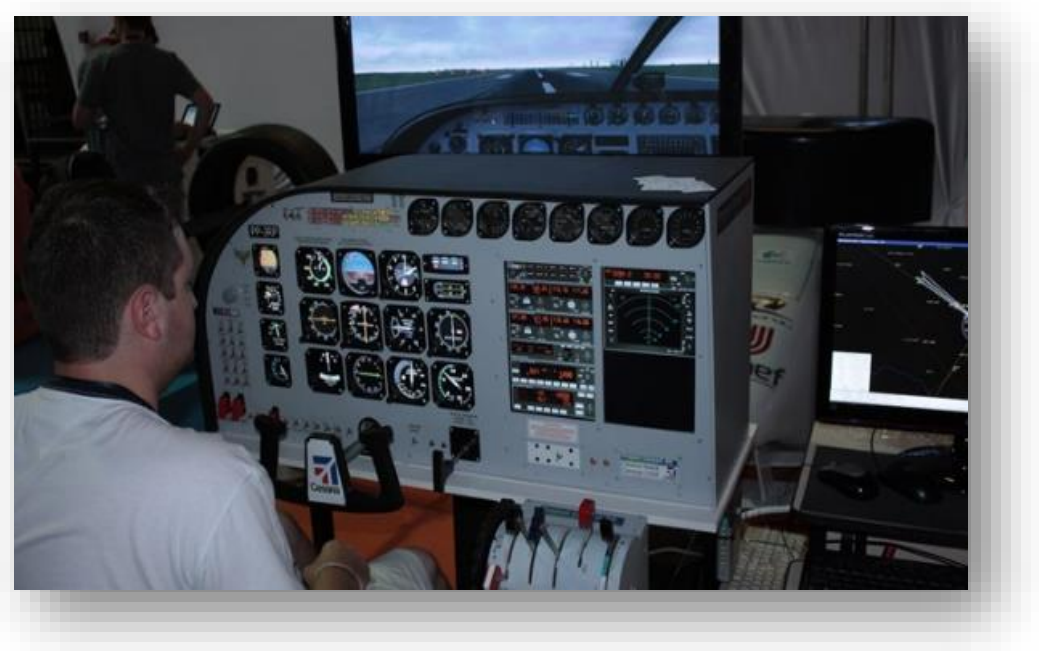

Figura 1 - Simulador de voo Fonte: Hamann (2012) 
Os recursos digitais permitem realizar diferentes práticas simuladas, tornando o processo formativo mais interessante e atrativo para os estudantes. Seguindo as tendências digitais e evolução da computação e dos recursos gráficos, a empresa 3D4Medical (THE, 2018) desenvolveu um modelo de Realidade Aumentada (RA) para estudo de Anatomia, chamado de Complete Anatomy (Anatomia Completa, em Português).

O Complete Anatomy é altamente detalhado e, segundo o site da 3D4Medical (THE, 2018), ele poderá auxiliar no ensino em Saúde substituindo, inclusive, a dissecção de cadáveres, facilitando a demonstração por parte dos professores de estruturas isoladas, ou ainda, se utilizado em clínicas, promover uma interação e explanação entre médico e paciente.

$\mathrm{Na}$ esfera da realidade virtual, como exemplo de simulador, pode ser citado o VISTLAB (figura 2), uma plataforma capaz de combinar elementos para um treinamento realista de equipe médica para estudos de Angiografia, simulando um procedimento de cateterismo (VIST-LAB, 2018).

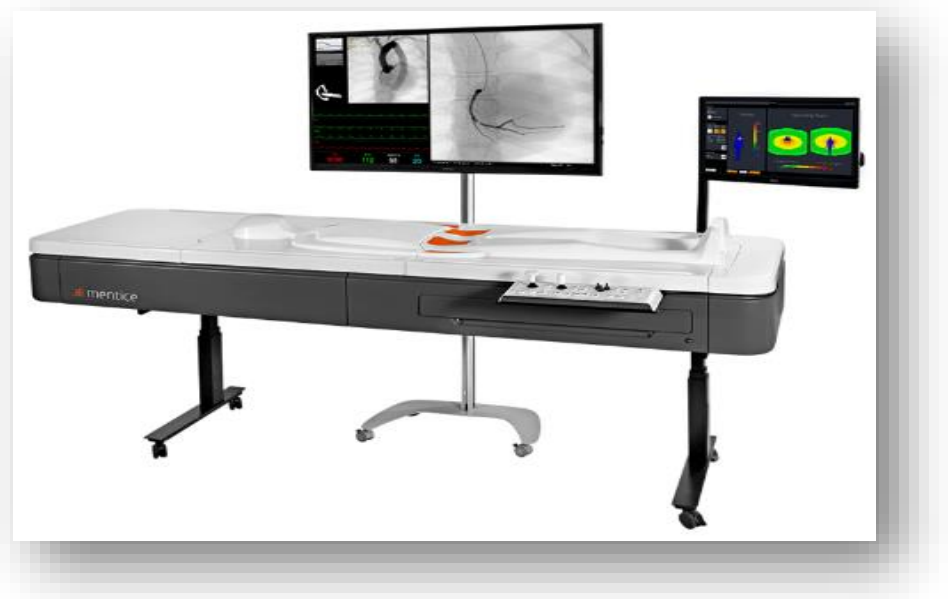

Figura 2 - Simulador para procedimentos de Angiografia Fonte: Mentice (2018)

Outro exemplo de objeto de aprendizagem digital é o da Pontifícia Universidade Católica (PUC) do Rio de Janeiro. A universidade foi a primeira da América Latina a utilizar a mesa virtual 3D, com o propósito do estudo da Anatomia Humana para os cursos de Medicina. A mesa permite uma análise bastante rica em detalhes, a exemplo do que ocorre com o Complete Anatomy, e uma das vantagens é a inserção de casos clínicos por meio de imagens de Tomografia Computadorizada e Ressonância Magnética, conforme relata Milene Couras (TV PUC-RIO, 2014), em entrevista. A figura 3 demonstra Milene Couras manipulando a mesa virtual. 


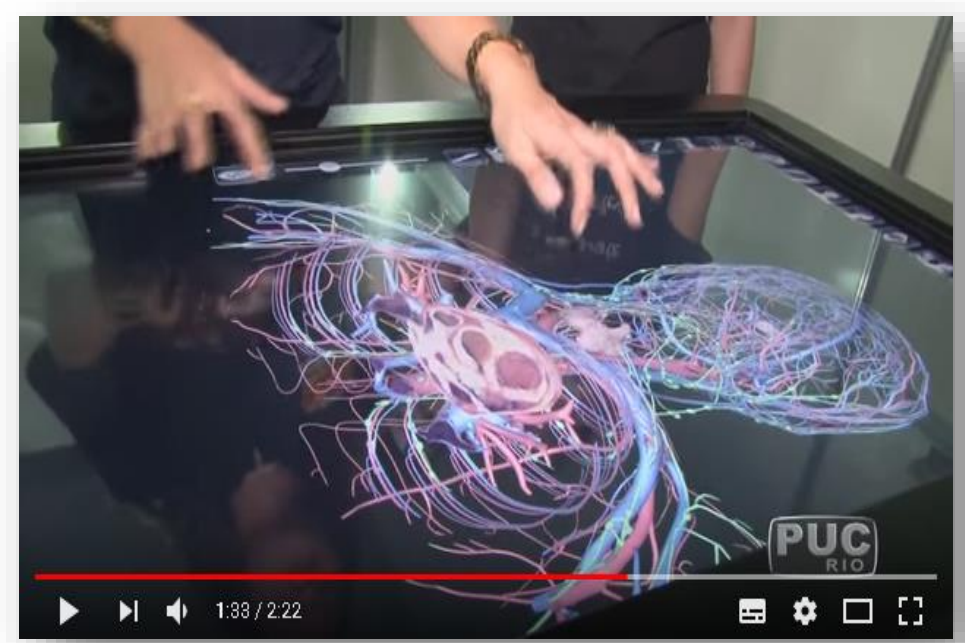

Figura 3 - Manipulação da mesa virtual em 3D Fonte: TV PUC-Rio (2014)

$\mathrm{Na}$ área da Saúde, especificamente, o uso de sistemas digitais utilizados para representar um paciente ou simular situações desponta como estratégia de grande potencial de auxílio docente. No estudo da Anatomia, por exemplo, nem todas as instituições de ensino dispõe de uma laboratório com peças anatômicas reais. Geralmente, o ensino se dá por meio de livros ou por meio de materiais sintéticos e, neste contexto, estudar por meio de imagens virtuais em 3D podem trazer ao estudante um entendimento mais amplo da Anatomia se comparada à aprendizagem apenas por atlas e livros. No campo prático da medicina ou enfermagem, o uso de processos simulados por meio digitais pode contribuir para proporcionar ao estudante um olhar panorâmico do que o aguarda em um campo de estágio ou no mercado de trabalho.

3.3 Aplicabilidade de softwares de simulação na Educação como um todo: trabalhos correlatos

Os softwares de simulação têm encontrado cada vez mais espaço em diferentes áreas educacionais, principalmente as que requerem algum tipo de treinamento prático de técnicas, como é o caso da formação na área da Saúde (MARAN; GLAVIN, 2003; ZIV et al., 2006; PAZIN FILHO; SCARPELINI, 2007; BALADEZ, 2009; SADIDEEN et al., 2012; LITTLEWOOD, 2013; FLOREZ; BEZ, 2014; CHETLEN, 2015; FERREIRA; CARVALHO J.; CARVALHO F., 2015; GEERAERTS; TRABOLD, 2016; CELESTINO, 2019).

Clayton e Gizelis (2005) criaram duas simulações aplicadas em um ambiente online com estudantes, para a aprendizagem de negociações e gestão de conflitos. Como resultados, o trabalho apresentou que: $67 \%$ dos estudantes concordaram que a simulação 
auxiliou no entendimento de conceitos teóricos; enquanto 33 \% não souberam afirmar; e $59 \%$ dos estudantes se prepararam mais para a atividade de simulação do que para os seminários tradicionais.

Borba (2014, p. 4) descreveu um exemplo de imersão visual, que ocorre por intermédio dos óculos 3D, que permitem uma maior vivência por meio de ilusões de óptica que promovem "uma relação de envolvimento, navegação, diálogo e, obviamente, imersão", sobretudo pelo detalhamento das interfaces gráficas. Um exemplo citado por Borba (2014 p. 6) é o sistema CAVE (Cavern Automatic Virtual Environment, Ambiente Virtual Automático da Caverna), no qual ocorre uma imersão ocular e motora, estimuladas por recursos audiovisuais.

Streicher et al. (2005) realizaram um experimento com uma turma de graduação em Engenharia Química, utilizando o ChemSep, um simulador de colunas de destilação, e consideraram que, em grande parte, a ferramenta foi vista pela maioria dos estudantes apenas como suporte para facilitar o procedimento, e que seria preciso um trabalho mais longo para que o software fosse percebido como ferramenta de aprendizagem.

Hillesheim e Schottz (2014) analisaram dois objetos simuladores disponíveis no Banco Internacional de Objetos Educacionais (BIOE). O primeiro se tratava de um microscópio virtual, capaz de analisar estruturas e funções das plantas, permitindo uma comparação e contextualização dos estudantes com a teoria. O segundo software representava o coração de uma ave, no qual os estudantes poderiam aprender sobre Anatomia e circulação cardíaca do animal, simulando ainda a retirada do pássaro de uma gaiola e realizando a cirurgia para a remoção do coração do animal.

Kampf e Stolero (2015) realizaram um estudo, envolvendo uma simulação por computador, acerca dos conflitos entre Israel e Palestina, por meio do game Peace Maker. Segundo os autores, este método pode auxiliar na percepção da óptica de ambas as partes frente ao mesmo conflito, o que colaboraria para a geração mútua da humanização e homogeneização do nível de conhecimento entre os usuários a respeito de um mesmo assunto.

Soares, Moraes e Oliveira (2015) realizaram um estudo de caso aplicando simuladores interativos em uma abordagem da Física Moderna e Contemporânea (FMC) para o ensino médio. A abordagem dos autores foi de cunho inicial e, também, de aprofundamento e contextualização de temas como: espectroscopia e a datação por carbono (14C); decaimento radioativo de radioisótopos; fissão nuclear e funcionamento de um reator nuclear.

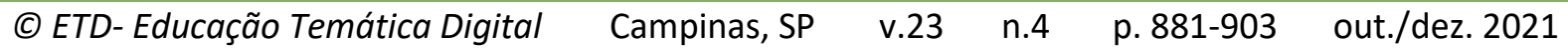


O quadro 2 apresenta alguns autores que abordaram o uso de softwares e simuladores em práticas de ensino-aprendizagem em diferentes áreas do conhecimento, dentre as quais: 1) Biológicas, na abordagem da Biologia e da Ecologia; 2) Engenharias, na abordagem das Engenharias de Produção, Ambiental e de Software; 3) Exatas, na abordagem de Matemática, de Física e de Química; 4) Humanas, no estudo da Língua Portuguesa, de Geografia, de Libras, de Psicologia, de Economia e Gestão e de História.

QUADRO 2. Uso de softwares e simuladores em diferentes práticas de ensino

Área

BIOLÓGICA Silva, Ferreira e Silva-Forsberg (2010); Alves et al., (2014); Gregório, Oliveira e $\mathrm{S}$

ENGENHARI Silva, Pinto e Subramanian (2007); De Alencar Carvalho, Carvalho e Da Silva AS

EXATAS Squire et al. (2004); Tavares (2015); Pinheiro, Pessoa Júnior e Araújo (2015).

HUMANAS Giraffa, Oliveira e Da Silva Borges (1999); De França, Da Silva (2014); Trindade et al. (2014); Leite (2015); Teixeira, Brito e Silva (2016).

Fonte: os autores (2020)

O quadro 3 apresenta algumas instituições que fazem o uso de softwares e/ou simuladores em processo de ensino-aprendizagem.

QUADRO 3. Instituições que utilizam softwares e/ou simuladores em práticas de ensino

\begin{tabular}{|c|l|l|}
\hline \multicolumn{1}{|c|}{ Nível de ensino } & \multicolumn{1}{|c|}{ Instituição } & \multicolumn{1}{|c|}{ Cidade/Estado } \\
\hline Cursos livres / Idiomas & Nova EAD (plataforma digital) & Online \\
\hline & Wizard & \multirow{2}{*}{ São Paulo, SP } \\
\hline Ensino Médio & Colégio VIP (Tremembé) \\
\hline & Colégio Palavra Viva & \\
\hline \multirow{2}{*}{ Educação Superior } & Colégio Dom Bosco & \\
\hline & Centro Universitário São Camilo & \\
\hline & Universidade Nove de Julho & Rio de Janeiro, RJ \\
\hline & Centro Universitário Senac & Jaguariúna, SP \\
\hline & Centro Universitário Augusto Mota & Florianópolis, SC \\
\hline
\end{tabular}

Fonte: os autores (2020) 
Na prática, é notável a aplicação versátil de softwares e simuladores na abordagem de diferentes conteúdos e níveis de ensino. Alguns exemplos são descritos a seguir:

- TerraView - ensino de Geografia para o ensino fundamental: promove socialização interativa dos resultados, por intermédio de mapas impressos (TRINDADE et al., 2014);

- Expressão Genética - Fundamentos - ensino de Genética e Hereditariedade, em Biologia, para o ensino médio: facilita o entendimento de conceitos abstratos, e por intermédio de um roteiro estruturado estimulam a discussão e o diálogo interpessoal após a observação do processo simulado (SILVA; FERREIRA; SILVA-FORSBERG, 2010);

- PhET: sugar-and-saltsolutions_pt_BR (Soluções de sal e açúcar) - simulador que aborda a Química para o ensino médio: melhora no rendimento dos alunos; abordagem de tópicos como concentração, dissolução, combinações de solutos, ligações iônica e covalente (PINHEIRO; PESSOA JÚNIOR; ARAÚJO, 2015; PHET, 2020);

- SPARSE - ensino de Engenharia de Software para graduação: aumento do conhecimento por meio de exercícios discursivos (SOUZA et al., 2010).

\subsection{Benefícios da simulação para o ensino-aprendizagem}

A simulação pode auxiliar na aprendizagem significativa, descrita por Ausubel e Novak (1980) como um processo de aquisição e desenvolvimento de novos conceitos que se relacionam com o conhecimento prévio do indivíduo. Assim, entende-se que a simulação e seus processos possam proporcionar maior retenção da aprendizagem quando comparada a outros métodos, aperfeiçoamento na aprendizagem de profissionais da área da Saúde e participação dos alunos para a problematização (FERREIRA; CARVALHO J.; CARVALHO, F., 2015). O quadro 4 apresenta benefícios sobre o uso de simuladores, relatados por diferentes autores em ordem cronológica crescente de publicação. 
QUADRO 4. Benefícios do uso de simuladores na Educação

\begin{tabular}{|c|c|}
\hline Autores & Conceitos \\
\hline Valente (1993) & $\begin{array}{l}\text { Aumento da concentração e foco nas atividades; } \\
\text { Feedback; } \\
\text { Teste de hipóteses sem prejuízos; } \\
\text { Melhora na compreensão de conceitos abstratos. }\end{array}$ \\
\hline $\begin{array}{l}\text { Bertsche, Crawford e } \\
\text { Macadam (1996) }\end{array}$ & $\begin{array}{l}\text { Demonstra a possibilidade de influências externas e a necessidade de } \\
\text { intervenções imediatas; } \\
\text { Percepção do impacto ocasionado pela comunicação e trabalho em equipe; } \\
\text { Observar o trabalho habitual e testar novas variações, abordagens ou } \\
\text { técnicas; } \\
\text { Coordenação de diversas pessoas na tomada de decisões. }\end{array}$ \\
\hline Cantero (2000) & $\begin{array}{l}\text { Comunicação direta com um mentor ou tutor, e a base de conhecimento } \\
\text { adaptativa e expansível; } \\
\text { Uso em diferentes plataformas integradas. }\end{array}$ \\
\hline Maran e Glavin (2003) & $\begin{array}{l}\text { Redução de riscos aos pacientes; } \\
\text { Criação de diversos cenários e situações; } \\
\text { Repetição da prática; } \\
\text { Aumento da retenção e precisão da aprendizagem; } \\
\text { Trabalho e aprendizagem de forma colaborativa. }\end{array}$ \\
\hline $\begin{array}{c}\text { Clayton e Gizelis } \\
(2005)\end{array}$ & $\begin{array}{l}\text { Amplitude e profundidade da aprendizagem; } \\
\text { Engajamento de pares (estudantes e professores); } \\
\text { Aumento na participação e preparo para exercícios simulados; } \\
\text { Desenvolvimento e transferência de habilidades. }\end{array}$ \\
\hline Ziv et al. (2006) & $\begin{array}{l}\text { Aprendizagem sem prejuízo aos pacientes; } \\
\text { Apresentação de contextos diferentes e complexos aos estudantes; } \\
\text { Possibilidade de erro e falha no decorrer da prática simulada; } \\
\text { Reduz a necessidade de animais vivos para servirem de modelos. }\end{array}$ \\
\hline Baladez (2009) & $\begin{array}{l}\text { Economia de tempo e dinheiro das forças armadas; melhor preparado de } \\
\text { novos soldados no quesito habilidades; } \\
\text { Desenvolvimento da capacidade de trabalhar em grupo; } \\
\text { Treinamento médico; } \\
\text { Possibilidade de repetições exaustivas e desenvolvimento de habilidades. }\end{array}$ \\
\hline Sadideen et al. (2012) & $\begin{array}{l}\text { Uso na educação cirúrgica; } \\
\text { Desenvolvimento de competências e expertise; } \\
\text { Desenvolvimento cognitivo e treinamento emocional; } \\
\text { Segurança para o desenvolvimento de treinamento e práticas deliberadas. }\end{array}$ \\
\hline Littlewood (2013) & $\begin{array}{l}\text { Aprendizagem centrada e personalizada para o aluno; } \\
\text { Segurança do paciente; } \\
\text { Aprendizagem por competências; } \\
\text { Trabalho colaborativo. }\end{array}$ \\
\hline
\end{tabular}

Fonte: os autores (2018)

Aumento da concentração e foco nas atividades;

Melhora na compreensão de conceitos abstratos.

Demonstra a possibilidade de influências externas e a necessidade de intervenções imediatas;

Observar o trabalho habitual e testar novas variações, abordagens ou

Comunicação direta com um mentor ou tutor, e a base de conhecimento Uaptativa e expansivel

Redução de riscos aos pacientes;

Criação de diversos cenários e situações;

Aumento da retenção e precisão da aprendizagem;

rabalho e aprendizagem de forma colaborativa.

Engajamento de pares (estudantes e professores);

Aumento na participação e preparo para exercícios simulados;

Apresentação de contextos diferentes e complexos aos estudantes;

Possibilidade de erro e falha no decorrer da prática simulada

Reduz a necessidade de animais vivos para servirem de modelos.

novos soldados no quesito habilidades;

Desenvolvimento da capacidade de trabalhar em grupo;

Uso na educação cirúrgica;

Desenvolvimento de competências expertise;

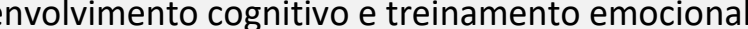

Aprendizagem centrada e personalizada para o aluno

Aprendizagem por competências; 
Qualquer diferença entre prática e simulação deve ser bastante esclarecida aos estudantes (MARAN; GLAVIN, 2003), de modo que o discente entenda que, apesar da aproximação e da similaridade, a simulação não é a realidade. No caso da Saúde, apesar dos benefícios que envolvem as práticas simuladas no ensino da medicina, as prática simuladas não substituem o contato direto com o paciente, entendido como momento fundamental para o desenvolvimento e entendimento da prática real (ZIV et al., 2006).

Um aspecto negativo da prática simulada é que esta pode gerar competitividade dentro da sala de aula, necessitando da mediação e percepção docente para a resolução de conflitos e, além disso, as simulações podem não funcionar em todas as práticas (CLAYTON; GIZELIS, 2005). Assim, é importante identificar o tipo de negócio ou contexto a que se pretende aplicar o método de simulação, avaliando as vantagens e benefícios reais que o método poderá trazer à prática (BERTSCHE; CRAWFORD; MACADAM, 1996).

Outros pontos relevantes, que podem ser vistos como uma oportunidade de desenvolvimento, são: complexidade do uso dos simuladores, a falta de intimidade com recursos tecnológicos e de informática, conteúdos extensos, que requeiram muitas simulações e a heterogeneidade das interfaces, o que ocasiona a necessidade constante de adaptação no uso dos simuladores por parte dos envolvidos (FLORES; BEZ BRUNO, 2014).

O uso da simulação ainda tem alguns obstáculos, pois requer preparo e conhecimento por parte dos professores para aplicação da metodologia, de modo que a formação e o preparo docente são pontos importantes para a aplicação das práticas simuladas, sobretudo quando relacionadas aos objetos digitais, que já fazem parte da realidade da sociedade de um modo geral (HILLESHEIM; SCHOTTZ, 2014).

De qualquer maneira, o futuro da simulação está assegurado, pois sempre será importante analisar um sistema antes de sua implantação ou aperfeiçoar um já existente, compreendendo melhor seu funcionamento, reduzindo custos e riscos. Assim, espera-se que a ciência venha a se desenvolver cada vez mais, e os simuladores serão cada vez mais presentes e vistos como a melhor solução para alcançar os resultados esperados (BALADEZ, 2009).

\section{DISCUSSÃO}

Os trabalhos analisados corroboram com as proposições de Baladez (2009) e Cantero (2000), que afirmam que os principais interesses e objetivos das aplicações das simulações e dos simuladores são o treinamento e a Educação (BALADEZ, 2009) e que podem abranger diferentes níveis educacionais (CANTERO, 2000). 
Os respondentes descritos no trabalho de Clayton e Gizelis (2005) escolheram o módulo de simulação como favorito, sendo considerado como um método útil no engajamento dos envolvidos. Isso converge com a perspectiva chamada de Design Centralizado no Usuário, na qual são considerados os aspectos como motivação do estudante e adaptação, envolvendo processos de gamificação (gamification), como referem Soloway e Pryor (1996) ou ainda a aprendizagem centrada e personalizada, de acordo com Littlewood (2013).

Após o uso de um software, os estudantes referidos no trabalho de Streicher et al. (2005) escreviam a respeito da experiência para consolidar a aprendizagem, mediante a percepção de que o conhecimento só seria efetivado e consolidado frente à certeza das respostas corretas, o que levou os autores a perceberem a insegurança dos alunos. Tal resultado difere do de Littlewood et al. (2013), sobre paciente realístico, em que a avaliação dos participantes também se deu por meio de perguntas, com resultados positivos e homogêneos, caracterizando a boa assimilação do conteúdo por parte de todos os estudantes envolvidos no processo.

Neste ponto, infere-se que um software nem sempre deva ser utilizado como único recurso ou objeto de aprendizagem, mas sim, parte de uma estratégia complementar e de suporte ao docente, pois pode apresentar múltiplas abordagens para diferentes níveis de compreensão. Também se entende que os resultados dependerão do tipo e estrutura do software a ser aplicado e da mediação pedagógica, como referem Bergeron (2006), a respeito da padronização e da interface amigável, e Ferreira, Carvalho J. e Carvalho F. (2015), em que o docente pode ser visto como a figura que redireciona as habilidades e competências dos estudantes.

Hillesheim e Schottz (2014) consideraram que existe uma grande aplicabilidade para os softwares enquanto objeto de aprendizagem no campo da simulação, principalmente por existir inúmeros tipos distribuídos gratuitamente, facilitando a sua inserção em qualquer escola que disponha de computadores. Desta forma, por tratar-se de uma ferramenta de baixo custo, sendo possível aproveitar a estrutura prévia de um laboratório de informática, por exemplo, reforça-se a ideia de que os softwares podem ser adaptados e aplicados em diferentes contextos e níveis educacionais, como referem Clayton e Gizelis (2005) e outros citados anteriormente.

No trabalho de Kampf e Stolero (2015), o uso do game Peace Maker (PEACEMAKER, 2018) promoveu aquisição de conhecimento sobre o assunto por todos os jogadores e, de certa forma, deixou todos os participantes em um patamar mais equilibrado e homogêneo para discussão do tema. Isso evidencia a possibilidade do uso de softwares para a discussão dos assuntos mais variados, com uma abordagem e discussão amplas a respeito de questões 
diversas como, por exemplo, raciais e sociais.

De acordo com Soares, Moraes e Oliveira (2015), em seu trabalho houve a interação, atenção dos estudantes e discussão de ideias, contribuindo para o maior envolvimento e participação dos alunos, para a melhora da interação dos participantes e para o envolvimento mais aprofundado sobre o tema. Isto reforça a questão do engajamento, da amplitude e do aprofundamento da aprendizagem, abordados por Clayton e Gizelis (2005), evidenciando o uso de softwares ou simuladores como uma estratégia atrativa para os estudantes que vivenciam uma era tecnológica e digital.

\section{CONCLUSÃO}

Este trabalho revisou a aplicabilidade e os benefícios de objetos e processos de simulação no campo educacional geral. A literatura analisada evidenciou a simulação, e seus processos, simuladores e softwares de simulação, como recursos preparatórios e interativos, capazes de atuar com ênfase na repetição segura de processos, a fim de desenvolver competência e habilidades em diferentes áreas e níveis educacionais.

Sobre o conceito de simulação, esta pode ser compreendida como uma técnica de ensino versátil e complementar, que se utiliza de recursos físicos ou digitais, para a apropriação e desenvolvimento de competências e habilidades em diferentes contextos educativos, permeada por uma estratégia de imitação e repetição prática sem prejuízos ou custos adicionais.

Conclui-se que a aplicabilidade da simulação, sobretudo por intermédio de softwares, pode corroborar para o preparo dos estudantes de diferentes áreas e níveis educacionais, tanto para o momento do estágio, quanto para a prática em mercado de trabalho, reduzindo inseguranças, promovendo o desenvolvimento de habilidades e, consequentemente, autoconfiança, bem como permitir ao docente intervir no decorrer do processo da aprendizagem, quando necessário, para correção de falhas.

\section{REFERÊNCIAS}

AEROCLUB DE BAURU. Simuladores de voo. 2018. Disponível em:

http://www.aeroclubebauru.com.br/escola-de-aviacao/simuladores-de-voo/ - Acesso em: 23 fev. 2018.

AEROCON. Simuladores de voo. 2018. Disponível em:

http://www.aerocon.com.br/Cursos-Simuladores-de-Voo.aspx - Acesso em: 23 fev. 2018. 
ALVES, Gabriel, et al. ControlHarvest: ensino de ecologia por meio de gamificação do controle biológico. BRAZILIAN SYMPOSIUM ON COMPUTERS IN EDUCATION = SIMPÓSIO BRASILEIRO DE INFORMÁTICA NA EDUCAÇÃO-SBIE, 2014. p. 342. Disponível em: https://www.researchgate.net/publication/275272463 ControlHarvest Ensino de Ecolo gia por Meio de Gamificacao do Controle Biologico - Acesso em: 13 abr. 2020.

ANDRADE, Antonio Manuel Valente. Recurso a simuladores na aprendizagem de fatores de segurança na exploração de Tecnologias da Informação. In: CARVALHO, A. A.

A. Aprender na era digital-jogos e mobile learning. Santo Tirso: De Facto, 2012. Cap. 3, p. 65-82. Disponível em:

http://www.diaadiaeducacao.pr.gov.br/portals/cadernospde/pdebusca/producoes pde/2 016/2016 pdp cien unicentro lucineiazampier.pdf - Acesso em: 11 nov. 2019.

ARAÚJO, Saionara; SILVEIRA, Daniela; MATOS, Pablo. Avaliação do software educacional eSinais no ensino-aprendizagem da língua portuguesa escrita e da LIBRAS. In: WORKSHOP DE INFORMÁTICA NA ESCOLA, 2017. Anais...2017. p. 323. Disponível em: http://pablofmatos.pro.br/files/paper/2017-WIE-AraujoEtAl.pdf Acesso em: 13 abr. 2020.

AUSUBEL, David P.; NOVAK, Joseph D.; HANESIAN, Helen. Psicologia educacional. Tradução Eva Nick. Rio de Janeiro: Interamericana, 1980.

CELESTINO, Marcelo Salvador. Aplicabilidade de softwares de simulação para o ensino de tomografia computadorizada para técnicos e tecnólogos em radiologia. Dissertação (Mestrado em Mídia e Tecnologia) - Universidade Estadual Paulista "Júlio de Mesquita Filho". Bauru, 2019. Disponível em: https://repositorio.unesp.br/handle/11449/181423 Acesso em: 14 abr. 2020.

BALADEZ, Fabio. O passado, o presente e o futuro dos simuladores. FaSCi-Tech, v. 1, n. 1, 2009. Disponível em:

https://www.fatecsaocaetano.edu.br/fascitech/index.php/fascitech/article/view/4/4 . Acesso em: 11 nov. 2019.

BAUDRILLARD, Jean. Simulacros e simulação. Tradução de Maria João da Costa Pereira. Lisboa: Relógio d'Água, 1991.

BERBEL, Neusi Aparecida Navas. As metodologias ativas e a promoção da autonomia de estudantes. Semina: Ciências Sociais e Humanas, Londrina, v. 32, n. 1, p. 25-40, jan./jun. 2011.

BERGERON, Bryan. Developing serious games. Boston: Charles River Media, 2006.

BERTSCHE, Dory; CRAWFORD, Christopher; MACADAM, Stephen E. Is simulation better than experience? The McKinsey Quarterly, n. 1, p. 50-58, 1996.

BIBLIOTECA VIRTUAL EM SAÚDE (BVS). Descritores em Ciências da Saúde (DeCS). 2018. Disponível em: http://decs.bvs.br/ - Acesso em: 11 nov. 2019. 
BORBA, Eduardo Zille. Imersão visual e corporal: paradigmas da percepção em simuladores. In: SOSTER, Demétrio de Azeredo; PICCININ, Fabiana. (Org.). Narrativas Comunicacionais Complexificadas II - A Forma. SantaCruz do Sul: Edunisc, 2014. p. 239256.

CAETANO, Diego Vaz; CARDOSO, Josué Dias; FERREIRA, Luana Guimarães Piani. 2017. SimEvolution: uma perspectiva de apoio ao aprendizado de biologia no ensino médio através de simulador educativo. Monografia (Tecnologia em Sistemas) - Centro Federal de Educação Tecnológica Celso Suckow da Fonseca (CEFET/RJ), Rio de Janeiro, RJ, 2017. Disponível em: https://bit.ly/2XxYWZO - Acesso em: 13 abr. 2020.

CANTERO, Manoel Ortega. Computers in education: The near future. In: Computers and Education in the 21st Century. Springer, Dordrecht, 2000. p. 3-16.

CARVALHO, Carlos Vitor de Alencar; CARVALHO, Janaina Veiga; SILVA, Júlio César da. VAPD-2D: Simulador para apoio ao ensino de engenharia ambiental. Revista Eletrônica TECCEN, 2008, v. 1, n. 2, p. 1-8. Disponível em: https://bit.ly/2wFdewC. Acesso em: 13 abr. 2020.

CHETLEN, Alison L., et al. Conventional medical education and the history of simulation in radiology. Academic radiology, v. 22, n. 10, p. 1252-1267, 2015.

CLAYTON, Govinda; GIZELIS, Theodora-Ismene. Learning through simulation or simulated learning? An investigation into the effectiveness of simulations as a teaching tool in higher education. 2005. Disponível em:

https://www.semanticscholar.org/paper/Learning-through-Simulation-or-SimulatedLearning-a-Clayton/c26e917501278fe52e864cf19b91f5cf283b184d . Acesso em: 11 nov. 2019.

EJ ESCOLA DE AVIAÇÃO CIVIL. Escola de voo, treinamento inicial e padronização de voo por instrumentos. Disponível em: https://www.ej.com.br/showinformativo/364/simuladores-de-voo-treinamento-inicial-e-padronizao-de-voo-porinstrumentos. Acesso em: 11 nov. 2019.

FERREIRA, Claudenice; CARVALHO, Josiane Martins; CARVALHO, Fernando Luís de Queiroz. Impacto da metodologia de simulação realística, enquanto tecnologia aplicada a educação nos cursos de saúde. Anais do Seminário Tecnologias Aplicadas a Educação e Saúde, v. 1, n. 1, 2015. Disponível em: https://bit.ly/2NBZTtk. Acesso em: 11 nov. 2019.

FITZSIMONS, M. Engaging Students' Learning through Active Learning. Irish Journal of Academic Practice, 2014, v. 3, n. 1, p. 1-26. Disponível em: https://bit.ly/2wFIFrM . Acesso em: 13 abr. 2020.

FLORES, Cecília Dias; BEZ, Marta Rosecler; BRUNO, Rosana Mussoi. O uso de simuladores no ensino da medicina. Revista Brasileira de Informática na Educação, v. 22, n. 02, p. 98, 2014. Disponível em: https://bit.ly/33hrp62 . Acesso em: 11 nov. 2019.

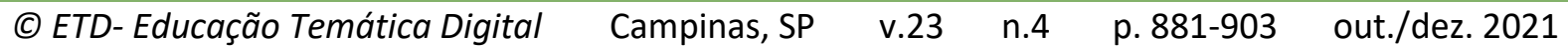


FRANÇA, Rozelma Soares de; SILVA, Ana Cristina Barbosa da. Avaliação de softwares educativos para o ensino de língua portuguesa. Revista Brasileira de Informática na Educação, 2014, v. 22, n. 3, p. 23-34. Disponível em: https://bit.ly/2RAxQgB . Acesso em: 13 abr. 2020.

FREGONEZE, Gisleine Bartolomei et al. Metodologia científica. Londrina: Educacional, 2014.

GEERAERTS, Thomas; TRABOLD, Fabien. The simulator of critical situations in anesthesia. In: FAUQUET-ALEKHINE, Philippe.; PEHUET, Nane. (Ed.). Simulation training: fundamentals and applications. Springer, 2016. Cap. 4, p. 87-94. Disponível em: https://bit.ly/36wuWzm . Acesso em: 11 nov. 2019.

GIRAFFA, Lucia Maria Martins. Uma odisséia no ciberespaço: O software educacional dos tutoriais aos mundos virtuais. Revista Brasileira de Informática na Educação, 2009, v. 17, n. 1, p. 20-30. Disponível em: https://bit.ly/2V2YalQ . Acesso em: 13 abr. 2020.

GREGÓRIO, Eliana Aparecida; OLIVEIRA, Luiza Gabriela; MATOS, Santer Alvares. Uso de simuladores como ferramenta no ensino de conceitos abstratos de Biologia: uma proposição investigativa para o ensino de síntese proteíca. Experiências em ensino de Ciências, 2016, v. 11, n. 1, p. 101-125. Disponível em: https://bit.ly/3cg2uE4 . Acesso em: 13 abr. 2020.

HAMANN, R. Project Cockpit BR: um simulador de voo incrível e 100\% nacional. 2012. Disponível em: https://www.tecmundo.com.br/campus-party-brasil-2012/19138-projectcockpit-br-um-simulador-de-voo-incrivel-e-100-nacional.htm . Acesso em: 11 nov. 2017.

HILLESHEIM, Geraldo Jose; SCHOTTZ, Eliane de Souza. Softwares simuladores interativos aplicados ao estudo de biologia. Maiêutica-Ciências Biológicas, v. 1, n. 1, 2014. Disponível em: https://bit.ly/2JQPk4t . Acesso em: 11 nov. 2019.

JESUS, Pedro Leandro Barreto de. Simuladores como ferramentas auxiliadoras no processo de ensino-aprendizagem de evolução biológica. 2018. Monografia (Licenciatura em Ciências Biológicas), Universidade Federal de Sergipe, São Cristóvão, 2018. Disponível em: https://bit.ly/2wAL59X . Acesso em: 13 abr. 2020.

KAMPF, Ronit; STOLERO, Nathan. Computerized simulation of the Israeli-Palestinian conflict, knowledge gap, and news media use. Information, Communication \& Society, $v$. 18, n. 6, p. 644-658, 2015.

LAW, Averill M.; KELTON, W. David. Simulation modeling and analysis. 2 ed. Singapore: Mc-Graw Hill International Editions, 1991.

LEITE, Daniela Sofia Teixeira. Jogos e simuladores: adequabilidade pedagógica no ensino da Economia e da Gestão: estudo do caso português. 2015. Dissertação (Mestrado em Gestão), Universidade Católica Portuguesa, Lisboa, Portugal. 2015. Disponível em: https://bit.ly/34yF3mK . Acesso em: 13 abr. 2020. 
LITTLEWOOD, Keith E. et al. High-fidelity simulation is superior to case-based discussion in teaching the management of shock. Medical teacher, v. 35, n. 3, p. e1003-e1010, 2013.

MANOVICH, Lev. Software takes command. New York: Bloomsbury Academic, 2013.

MARAN, Nikki J.; GLAVIN, Ronnie J. Low-to high-fidelity simulation-a continuum of medical education? Medical education, v. 37, n. S1, p. 22-28, 2003.

McHANEY, Roger. Understanding computer simulation. BookBoon, 2009. Disponível em: https://bit.ly/2PNzj3k . Acesso em: 11 nov. 2019.

PASSOS, Denis da Silva; VENEGA, Virginia de Sousa; ROCHA, Marcelo Lisboa. Softwares para suporte no ensino de engenharia civil: um mapeamento sistemático do uso nas instituições brasileiras. REVISTA CEREUS, 2017, v. 9, n .4, p. 2-18. Disponível em: https://www.researchgate.net/publication/323686502 SOFTWARES PARA SUPORTE NO ENSINO DE ENGENHARIA CIVIL UM MAPEAMENTO SISTEMATICO DOS SEUS USOS NAS INSTITUICOES BRASILEIRAS. Acesso em: 13 abr. 2020.

PAZIN FILHO, Antônio.; SCARPELINI, Sandro. Simulação: definição. Medicina (Ribeirão Preto. Online), v. 40, n. 2, p. 162-166, 2007. Disponível em: https://bit.ly/36wOmnL . Acesso em 11 nov. 2019.

PEACEMAKER: Play the news. Solve the puzzle. Impact Games, 2018. Disponível em: http://www.peacemakergame.com/ . Acesso em: 11 nov. 2019.

PHET. Soluções de Açúcar e Sal. 2020. Disponível em:

https://phet.colorado.edu/pt BR/simulation/sugar-and-salt-solutions . Acesso em: 20 abr. 2020.

PINHEIRO, A. F.; PESSOA JÚNIOR, E. S. F.; ARAÚJO, M. D. Software de simulação: um recurso facilitador no processo de ensino aprendizagem de química no ensino médio. In: CONGRESSO NACIONAL DE EDUCAÇÃO - EDUCER, 12., 2015, Curitiba, PR. Anais... Curitiba, PR, 2015, p.2042-2057. Disponível em: https://bit.ly/2V4BSA3 . Acesso em: 13 abr. 2020.

PORTAL de periódicos. CAPES/MEC. 2017. Disponível em: https://www.periodicos.capes.gov.br/ . Acesso em: 11 nov. 2019.

PRENSKY, Marc. Aprendizagem baseada em jogos digitais. São Paulo: SENAC, p. 575, 2012.

PRENSKY, Marc. Digital natives, digital immigrants. On the Horizon, v. 9, n. 5, p. 1-6, 2001. Disponível em: http://www.marcprensky.com/. Acesso em: 11 nov. 2019.

PUBMED. National Center for Biotechnology Information (NCBI). 2017. Disponível em: https://www.ncbi.nlm.nih.gov/pubmed/. Acesso em: 11 nov. 2019. 
RODRIGUES, R.; SOARES, R. P.; SECCHI, A. R. Coleção de 10 problemas numéricos típicos em Engenharia Química resolvidos com o simulador EMSO. In: CONGRESSO BRASILEIRO DE ENGENHARIA QUÍMICA, 17., 2008. Anais..., 2008.

SADIDEEN, Hazim. et al. Simulators and the simulation environment: getting the balance right in simulation-based surgical education. International Journal of Surgery, v. 10, n. 9, p. 458-462, 2012. Disponível em: https://bit.ly/2CdQ9Ae . Acesso em: 11 nov. 2019.

SCIENCE DIRECT. Search for peer-reviewed journals, articles, book chapters and open access content. 2018. Disponível em: https://www.ScienceDirect.com . Acesso em: 11 nov. 2019.

SILVA, Karla Nunes da; FERREIRA, Luciana da Cunha; SILVA-FORSBERG, Maria Clara. Simulações computacionais aplicadas ao ensino da Biologia. In: SEMINÁRIO NACIONAL DE EDUCAÇÃO PROFISSIONAL E TECNOLÓGICA (SENEPT), 2., 2010. Anais... Disponível em: http://if.ufmt.br/eenci/artigos/Artigo ID303/v11 n1 a2016.pdf . Acesso em: 13 abr. 2020.

SILVA, Liane Márcia Freitas; PINTO, Marcel de Gois; SUBRAMANIAN, Anand. Utilizando o software Arena como ferramenta de apoio ao ensino em engenharia de produção. In: ENCONTRO NACIONAL DE ENGENHARIA DE PRODUÇÃO, 27., 2007. Anais... Disponível em: https://bit.ly/3b80jjO . Acesso em: 13 abr. 2020.

SOARES, Antonio Augusto; MORAES, Letícia Estevão; OLIVEIRA, Franciéle Gonçalves. Ensino de matéria e radiação no ensino médio com o auxílio de simuladores interativos. Caderno Brasileiro de Ensino de Física, v. 32, n. 3, p. 915-933, 2015.

SOLOWAY, Eliot; PRYOR, Amanda. The next generation in human computer interaction. Communications of the ACM, v. 39, n. 4, p. 16-18, 1996.

SOUZA, Mariane M., et al. SPARSE: Um ambiente de ensino e aprendizado de engenharia de software baseado em jogos e simulação. In: Brazilian Symposium on Computers in Education (Simpósio Brasileiro de Informática na Educação-SBIE), 2010. Disponível em: https://bit.ly/2V3LSJI . Acesso em: 13 abr. 2020.

SQUIRE, Kurt, et al. Electromagnetism supercharged! Learning physics with digital simulation games, 2004. p. 513-520. Disponível em: https://bit.ly/3b5PT6b . Acesso em: 13 abr. 2020.

STRAUBHAAR, Joseph; LaROSE, Robert. Comunicação, mídia e tecnologia. Tradução de José Antonio Lacerda Duarte. São Paulo: Pioneira Thompson Learning, 2004.

STREICHER, Samantha J. et al. Learning Through Simulation. Chemical Engineering Education, v. 39, n. 4, p. 288-295, 2005.

TAVARES, Marcelo Carvalho. $O$ uso dos softwares educativos no ensino-aprendizagem das quatro operações matemáticas. Ensino \& Pesquisa, 2015, v. 13, n. 01, p. 109-117. Disponível em: https://bit.ly/3baA2TS . Acesso em: 13 abr. 2020.

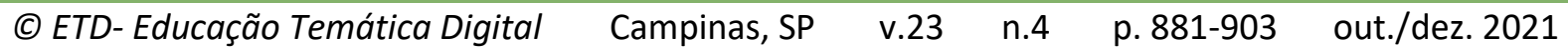


TEIXEIRA, Floripes; SILVA, Fábio; BRITO, Patrick. Uma revisão sistemática sobre softwares educacionais para o ensino de Libras. In: Brazilian Symposium on Computers in Education (Simpósio Brasileiro de Informática na Educação-SBIE), 2016. p. 896. Disponível em: https://bit.ly/2xpzbQB . Acesso em: 13 abr. 2020.

TRINDADE, Filipe Silveira, et al. Uso de softwares livres de SIG como uma ferramenta no ensino de geografia: mapeamento de áreas de risco. Caminhos de Geografia - revista online, 2014, v. 15, n. 51. Disponível em: https://bit.ly/3cikBt8 . Acesso em: 13 abr. 2020.

TV PUC-RIO. TV PUC-Rio: Mesa anatômica virtual 3D - Mostra PUC. [YouTube]. 2014. 2min22seg - 1min12seg. Disponível em: https://youtu.be/cuR5-N2a3co . Acesso em: 11 nov. 2019.

VALENTE, José Armando. Diferentes usos do computador na educação. In: (Org). Computadores e conhecimento: repensando a educação. 2. ed. Campinas: UNICAMP/NIED, 1993. Cap. 1, p. 1-28. Disponível em: https://bit.ly/2WPzzQM . Acesso em: 11 nov. 2019.

VIDAL-GOMEL, Christine; FAUQUET-ALEKHINE, Philippe. Reflections and Theoretical Contributions Regarding Trainers. In: FAUQUET-ALEKHINE, Philippe; PEHUET, Nane. (Ed.). Simulation training: fundamentals and applications. Springer, 2016. Cap. 1, p. 1-30. Disponível em: https://bit.ly/36wuWzm . Acesso em: 11 nov. 2019.

ZIV, Amitai. et al. Simulation-based medical education: an ethical imperative. Academic Medicine, v. 78, n. 8, p. 783-788, 2003. 